Published online 2017 April 13.

Abstract

\title{
Predictive Value of Transvaginal Ultrasound in Diagnosis of Endometriosis: A Three Year Study
}

\author{
Maryam Niknejadi, ${ }^{1,}{ }^{*}$ Maryam Javam, ${ }^{2}$ and Mohammad Chehrazi ${ }^{3}$ \\ ${ }^{1} \mathrm{MD}$, Radiologist, Department of Reproductive Imaging, Reproductive Biomedicine Research Center, Royan Institute for Reproductive Biomedicine, ACECR, Tehran, Iran \\ ${ }^{2}$ BSC, Department of Epidemiology and Reproductive Health, Reproductive Biomedicine Research Center, Royan Institute for Reproductive Biomedicine, ACECR, Tehran, Iran \\ ${ }^{3} \mathrm{PhD}$, Department of Epidemiology and reproductive health, Reproductive Biomedicine Research Center, Royan Institute for Reproductive Biomedicine, ACECR, Tehran, Iran \\ "Corresponding author: Maryam Niknejadi. E-mail: mniknezhady1390@gmail.com
}

Received 2016 December 21; Accepted 2017 February 08.

\begin{abstract}
Background: To assess the predictive value of various transvaginal sonography findings in characterizing endometriosis. Methods: This prospective investigation was conducted at Royan Institute, Tehran, Iran, from April 2010 till March 2013. All infertile women presenting with clinical symptoms of endometriosis, who were booked for laparoscopy, underwent a preoperative trasvaginal sonography by an expert radiologist. Endometriosis was diagnosed on TVS according to detecting one or more of following findings: echogenic points on serosal surface of the ovaries, ovarian endometrioma and signs of pelvic adhesion (blurred ovary margins, fixation of ovaries, deviation of ovaries or decreased distance between them). A logistic regression analysis was performed to identify prognostic factors in detection of presence of endometriosis. Each parameter was entered sequentially into the model based on hosmer-lemeshow algorithm. Values were calculated by Stata12 software regarding laparoscopy as gold standard.

Results: Totally, 101women were evaluated during the study. Endometriosis was present at laparoscopy in 58 patients. Mean age and the duration of infertility were calculated $30.78 \pm 4.79$ and $4.2 \pm 2.91$ years respectively. Frequencies of each sonographic finding were as follow: echogenic points (\%31.68), ovarian endometrioma (\%30.69), blurred ovary margin (\%7.92), fixation of ovaries (\%15.9), deviation of ovaries (\%21.8), and decreased distance between ovaries (\%8.9). As derived by logistic regression analysis, detection of "ovarian endometrioma", "fixation of ovaries" and "ovarian deviation" strongly predicts endometriosis (odds ratio of $42.27,46.89$ and 14.78 respectively).

Conclusions: Our study demonstrated that TVS has a reliable predictive value in diagnosis of endometriosis. "Ovarian endometrioma”, "deviation of ovaries" and "ovarian fixation" can be used as important criteria for radiologists and gynecologists.
\end{abstract}

Keywords: Transvaginal Sonography (TVS), Laparoscopy, Endometriosis

This is an abstract presented in the 33rd Iranian congress of radiology (ICR) and the 15th congress of Iranian radiographic science association (IRSA). 